\title{
Long noncoding RNA IRAIN acts as tumor suppressor via miR-125b in multiple myeloma
}

\author{
YANXIA JIANG, JIAN CHEN and GUOAN CHEN
}

\begin{abstract}
Hematology Department, The First Affiliated Hospital of Nanchang University, Nanchang, Jiangxi 330000, P.R. China
\end{abstract}
Received January 30, 2018; Accepted September 7, 2019

DOI: $10.3892 / \mathrm{ol} .2019 .11012$

\begin{abstract}
Long noncoding RNAs (lncRNAs) are aberrantly expressed in a variety of cancer types. The lncRNA IGF1R antisense imprinted non-protein coding RNA (IRAIN) is associated with various cancer types, yet the role of IRAIN in multiple myeloma (MM) progression remains unclear. In the present study it was identified that IRAIN may act as a tumor suppressor in MM, whilst microRNA (miR)-125b may promote tumorigenesis. Downregulation of IRAIN significantly increased the expression of miR-125b. Furthermore, by using dual-luciferase reporter assays, IRAIN was identified as a target of miR-125b. Knockdown of IRAIN promoted MM cell proliferation in vitro. Thus, expression levels of IRAIN may be used to predict the clinical prognosis of patients with MM and may be a novel therapeutic target for treating MM.
\end{abstract}

\section{Introduction}

Multiple myeloma (MM) is a malignant plasma cell (PC) disorder characterized by the presence of malignant PCs within the bone marrow. MM accounts for $\sim 1 \%$ of neoplastic diseases and $13 \%$ of all hematological cancer types, with a 5 -year survival rate of only $40 \%$ (1). Although treatment strategies have evolved from traditional chemotherapy and autologous hematopoietic stem cell transplantation to novel targeted drug therapies, patient outcomes have not improved notably (2). Therefore, the need to investigate novel functional molecular and therapeutic targets for the treatment of MM is ever increasing.

Long noncoding RNAs (lncRNAs) are >200 nucleotides (nt) long and cannot be translated into proteins (3). Previous studies have demonstrated that IncRNAs are involved in tumor development and may be used as diagnostic markers

Correspondence to: Professor Guoan Chen, Hematology Department, The First Affiliated Hospital of Nanchang University, 17 Yongwaizheng Street, Donghu, Nanchang, Jiangxi 330000, P.R. China

E-mail: guoanchen010@sina.com

Key words: IGF1R antisense imprinted non-protein coding RNA, microRNA-125b, multiple myeloma, metastasis, cell proliferation, cell apoptosis, cell migration of cancers (4). Accumulating evidence has demonstrated that lncRNAs [colon cancer associated transcript 1, metastasis associated lung adenocarcinoma transcript 1 (MALAT1) and urothelial cancer associated 1 (UCA1)] serve a role in MM, suggesting their importance in MM progression (5-7). However, the function of lncRNAs in MM malignancy and tumorigenesis remains unclear.

The lncRNA IGF1R antisense imprinted non-protein coding RNA (IRAIN), which is 5,359 nt in length, is located on chromosome 15q26.3. Previous studies have suggested that IRAIN is downregulated in prostate cancer and blood obtained from high-risk acute myeloid leukemia (AML) patients $(8,9)$. Kang et al (10) reported that IRAIN also acts as a tumor suppressor in breast cancer. However, the functional role and underlying mechanisms of IRAIN in MM are poorly understood. In the present study, IRAIN expression was detected in MM tissues and cell lines and an initial analysis of its molecular mechanisms of action was performed. This present study provides novel insights into the function of IRAIN in MM development, and suggests that this lncRNA may serve a role in MM tumorigenesis.

\section{Materials and methods}

Sample collection. The specimens used in the present study were obtained from 35 patients who were diagnosed according to the National Comprehensive Cancer Network (NCCN) clinical practice guidelines for MM at the First Affiliated Hospital of Nanchang University (Nanchang, China), between October 2015 and May 2017. All patients who received chemotherapy and/or biotherapy were excluded and patients with other types of malignant tumors were eliminated. A total of 20 plasma samples in the validation set from healthy individuals were used as controls. Venous blood was collected in EDTA tubes (BD Biosciences, Franklin Lakes, NJ, USA). The plasma was transferred to a fresh tube and stored at $-80^{\circ} \mathrm{C}$ following snap-freezing in liquid nitrogen. The study was approved by the Research Ethics Committee of Nanchang University and written informed consent was obtained from all study subjects.

RNA isolation from human plasma and reverse transcription-quantitative polymerase chain reaction $(R T-q P C R)$. Total RNA was extracted from human plasma using the mirVana PARIS RNA Isolation kit (Ambion; 
Thermo Fisher Scientific, Inc., Waltham, MA, USA) following the manufacturer's protocol for liquid samples. The concentration and purity of extracted RNA were measured using 260 and $280 \mathrm{~nm}$ optical densities. cDNA was synthesized from RNA via RT using gene-specific primers (Applied Biosystems; Thermo Fisher Scientific, Inc.) using the Moloney Murine Leukemia Virus RT kit (GeneCopoeia, Inc., Rockville, MD, USA), according to the manufacturer's protocol: $42^{\circ} \mathrm{C}$ for $2-5 \mathrm{~min}, 42^{\circ} \mathrm{C}$ for $50 \mathrm{~min}$, and $70^{\circ} \mathrm{C}$ for $15 \mathrm{~min}$. To determine microRNA (miR)-125b expression levels, qPCR was performed using SYBR Green (Takara Bio, Inc., Otsu, Japan). U6 was used as the internal control. PCR conditions were as follows: 40 cycles of $95^{\circ} \mathrm{C}$ for $5 \mathrm{~min}, 95^{\circ} \mathrm{C}$ for $45 \mathrm{sec}, 55^{\circ} \mathrm{C}$ for $15 \mathrm{sec}$ and $72^{\circ} \mathrm{C}$ for $50 \mathrm{sec}$. Samples were analyzed in triplicate and gene expression was quantified by normalizing target gene expression to that of the internal control using the $2^{-\Delta \Delta \mathrm{Cq}}$ formula (11). The primer sequences used were as follows: IRAIN forward, 5'-CGACACATG GTCCAATCACTGTT-3' and reverse, 5'-AGACTCCCC TAGGACTGCCATCT-3'; miR-125b forward, 5'-TGCGCT AAAGTGCTTATAGTGC-3' and reverse, 5'-CCAGTGCAG GGTCCGAGGTATT-3'; and U6 forward, 5'-CTCGCTTCG GCAGCACA-3' and reverse, 5'-AACGCTTCACGAATT TGCGCTC-3'.

Cell lines and transfection. Human MM cell lines (MM.1S, U266 and RPMI-8226) and normal PCs (nPCs) were cultured in RPMI-1640 (Gibco; Thermo Fisher Scientific, Inc.) supplemented with $10 \%$ fetal bovine serum (FBS) (Gibco; Thermo Fisher Scientific, Inc.) and $1 \%$ penicillin/streptomycin at $37^{\circ} \mathrm{C}$ in a 5\% $\mathrm{CO}_{2}$-containing atmosphere. When the cells reached $80 \%$ confluence, cells in the logarithmic growth phase were collected. The cell lines were obtained from the American Type Culture Collection (Manassas, VA, USA). nPCs in the control group were extracted from the plasma of healthy volunteers and verified by flow cytometry (FITC-IgG, ab6755 and FITC-CD138, ab34164; Abcam, Cambridge, MA, USA) on a FACSCalibur flow cytometer (BD Biosciences). When the cells reached $70-80 \%$ confluence, they were transfected with IRAIN-targeting small interfering (si)RNA1, siRNA2, siRNA3 and negative control (NC) using riboFECT ${ }^{\mathrm{TM}}$ CP transfection reagents (Guangzhou RiboBio Co., Ltd., Guangzhou, China), according to the manufacturer's instructions. The sequences were: siRNA1 forward, 5'-GCGGGC ACAUACUCACUUUTT-3' and reverse, 5'-AAAGUGAGU AUGUGCCCGCTT-3'; siRNA2 forward, 5'-CCCUUAAUG UGGUCCGGUUTT-3' and reverse, 5'-AACCGGACCACA UUAAGGGTT-3'; siRNA3 forward, 5'-GAGCGACACUGC UUAUUAATT-3' and reverse, 5'-UUAAUAAGCAGUGUC GCUCTT-3'; si-NC forward, 5'-UUCUCCGAACGUGUC ACGUTT-3' and reverse, 5'-ACGUGACACGUUCGGAGA ATT-3'. miR-125b inhibitor was purchased from Shanghai GenePharma Co., Ltd. (Shanghai, China), with the following sequence: miR-125b inhibitor forward, 5'-UCACAAGUU AGGGUCUCAGGGA-3'. MiR-125b inhibitor was formulated in $0.9 \% \mathrm{NaCl}$ to a final concentration of $10 \mathrm{mg} / \mathrm{ml}$. The cells were subjected to RT-qPCR to measure the expression of IRAIN. As IRAIN siRNA3 was most effective at knocking down IRAIN expression, this siRNA was used for all subsequent experiments.
Cell proliferation and colony formation assays. For the cell proliferation assays, cells were plated in individual wells in a 96-well plate (1,500 cells/well) and examined $48 \mathrm{~h}$ post-transfection using a Cell Counting Kit-8 (CCK8; Dojindo Molecular Technologies, Inc., Kumamoto, Japan). Optical density values were determined at $450 \mathrm{~nm}$ wavelength using a microplate reader. For the survival rate assay, the numbers of viable cells were counted using Trypan blue dye and a Countstar Cell Counter (ALIT Life Sciences Co., Ltd., Shanghai, China), according to the manufacturer's protocol. All assays were performed in triplicate. For the colony formation assay, a total of 500 cells were plated in a six-well plate and maintained in medium containing $10 \%$ FBS, which was replaced every 5 days. After 2 weeks, the cells were fixed with methanol at $23^{\circ} \mathrm{C}$ for $1 \mathrm{~h}$ and stained with $0.1 \%$ crystal violet at $37^{\circ} \mathrm{C}$ for $30 \mathrm{~min}$. Visible colonies were manually counted by visual inspection. Triplicate wells were measured in each treatment group.

Apoptosis analysis. The cells in each group were collected at 24, 48 and $72 \mathrm{~h}$ following transfection and cold PBS was used to wash the cells three times. The cells were resuspended in $500 \mu \mathrm{l}$ pre-cooled binding buffer at a concentration of $5 \times 10^{6}$ cells $/ \mathrm{ml}$. A total of $100 \mu 1$ of the cell suspension was added to flow cytometry tubes and $5 \mu 1$ Annexin V-fluorescein isothiocyanate (Beyotime Institute of Biotechnology, Haimen, China) was added. Following mixing, the samples were incubated at room temperature in the dark for $15 \mathrm{~min}$ and at $5 \mathrm{~min}$ prior to the measurements, $5 \mu \mathrm{l}$ of $10 \mathrm{mg} / \mathrm{l}$ propidium iodide dye was added. Samples were immediately analyzed via fluorescent-activated cell sorting and BD FACSuite ${ }^{\mathrm{TM}}$ software (BD Biosciences), without washing or fixation. Each sample was analyzed three times.

Luciferase reporter analysis. The pGL3-IRAIN-3' untranslated region (UTR)-wild-type/mutated vector (Promega Corporation, Madison, WI, USA) was co-transfected with control plasmid or miR-125b-expressing plasmid into 293T cells (CRL-3216 ${ }^{\mathrm{TM}}$; American Type Culture Collection, Manassas, VA, USA) using Lipofectamine ${ }^{\circledR} 2000$ (Thermo Fisher Scientific, Inc.). Firefly and Renilla luciferase activities were measured consecutively $24 \mathrm{~h}$ after transfection using a Dual-Luciferase Reporter assay kit (Promega Corporation).

Prediction of miR targets. Computational prediction of $\mathrm{miR}$ targets was performed using the online database miRcode (www.mircode.org).

Statistical analysis. For data analysis, the SPSS 16.0 (SPSS, Inc., Chicago, IL, USA) and Microsoft Excel (Microsoft Office 2013 for Windows; Microsoft Corporation. Redmond, WA, USA) were used. Each experiment was performed at least three times and the values are reported as the mean \pm standard deviation. Differences between two groups were evaluated by Student's t-tests. For multiple-groups comparisons, one-way analysis of variance was used, followed by post hoc Newman-Keuls tests. $\mathrm{P}<0.05$ was considered to indicate a statistically significant difference. For Pearson's correlation coefficient analysis, GraphPad Prism software (version 5.01; GraphPad Sofware, Inc., La Jolla, CA, USA) was used. 

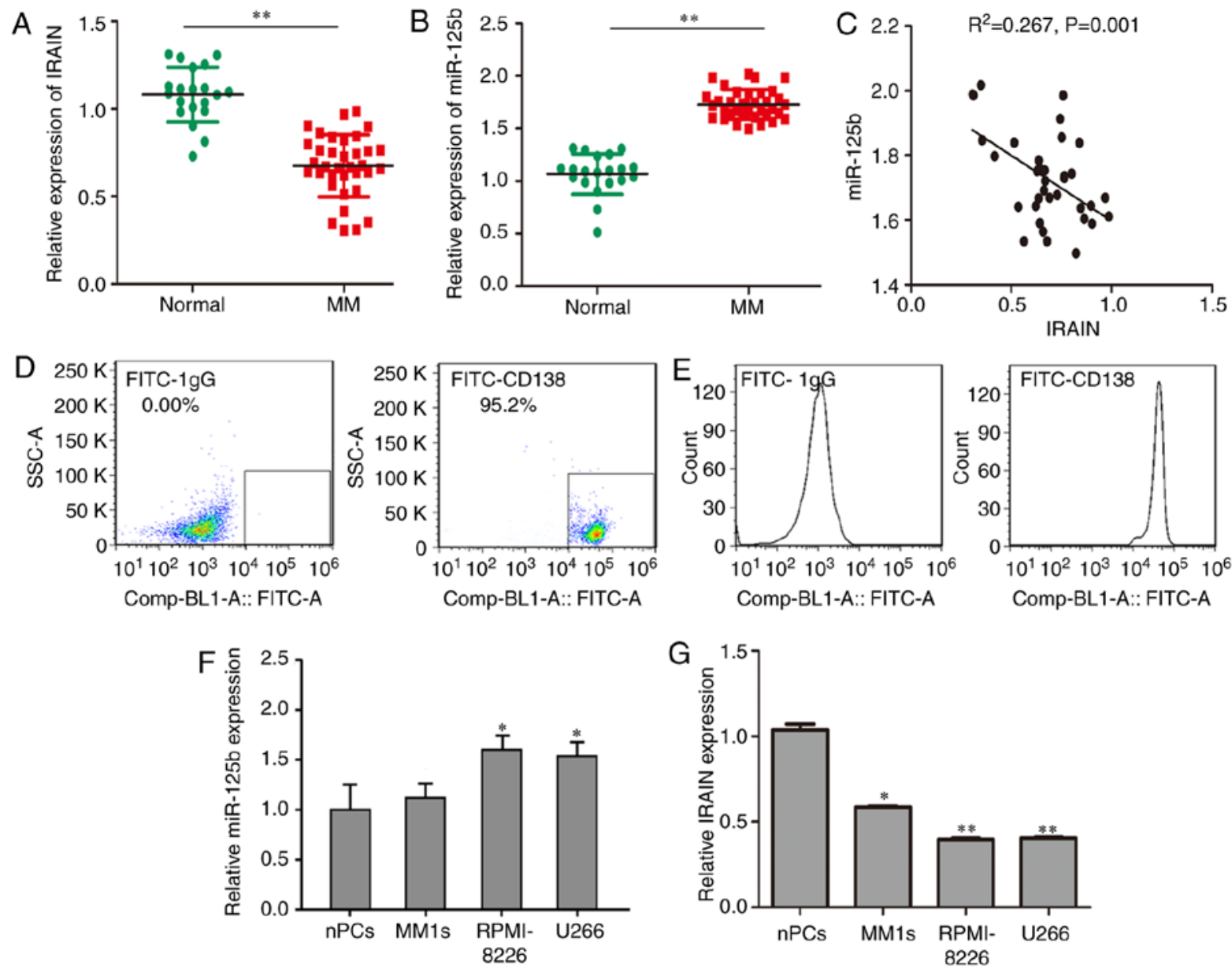

Figure 1. Determination of IRAIN and miR-125b expression in human MM cell lines and clinical samples via reverse transcriptase-quantitative polymerase chain reaction. (A) Relative expression levels of IRAIN in the plasma of patients with MM compared with healthy individuals. (B) Relative expression levels of miR-125b in the plasma of patients with MM compared with healthy individuals. (C) An inverse correlation between expression levels of miR-125b and IRAIN was observed in patients with MM. (D and E) Flow cytometry was applied to verify normal plasma cells. Data are presented as (D) dot plots and (E) histograms. (F) Expression levels of miR-125b in the MM cell lines MM.1S, U266 and RPMI-8226 compared with nPCs. (G) Expression levels of IRAIN in the MM cell lines MM.1S, U266 and RPMI-8226 compared with nPCs. Each sample was analyzed in triplicate and error bars represent the standard deviation. ${ }^{*} \mathrm{P}<0.05,{ }^{* * *} \mathrm{P}<0.01$ vs. nPCs. MM, multiple myeloma; nPCs, normal plasma cells; IRAIN, IGF1R antisense imprinted non-protein coding RNA; miR, micro-RNA; FITC, fluorescein isothiocyanate; $\mathrm{CD}$, cluster of differentiation.

\section{Results}

miR-125b is overexpressed and IRAIN is downregulated in plasma from patients with MM and in MM cell lines. RT-qPCR was performed in 35 plasma samples from patients with MM and 20 samples from healthy individuals. The expression level of IRAIN in patients with MM was significantly lower compared to that in healthy individuals (Fig. 1A). By contrast, compared with that in cells from healthy subjects, the level of circulating miR-125b was upregulated in cells from patients with MM. The normalized miR-125b expression levels in the patients with MM and healthy subjects were $1.69 \pm 0.03$ and $1.30 \pm 0.08$, respectively and miR-125b expression in MM patients was significantly higher compared with that in healthy individuals (Fig. 1B). An inverse correlation between miR-125b and IRAIN was also observed in patients with $\mathrm{MM}\left(\mathrm{P}<0.01 ; \mathrm{R}^{2}=0.267\right)$ (Fig. 1C). Normal plasma cells in the control group were cluster of differentiation 138-positive cells extracted from the plasma of healthy volunteers and verified by flow cytometry (Fig. 1D and E). Consistent with these data, the RPMI-8226 cell line expressed the highest level of miR-125b (Fig. 1F) and the lowest level of IRAIN (Fig. 1G).
Downregulation of IRAIN promotes cell proliferation. To investigate the biological role of IRAIN in MM cell lines, MM cells were transfected with IRAIN-targeting siRNA. IRAIN siRNA 3 possessed the most potent ability to knock down IRAIN (Fig. 2A). The CCK8 assay results demonstrated that the viability of U266 and RPMI-8226 cells was increased by transfection with si-IRAIN (Fig. 2B and C). Consistent with the CCK8 assay results, the colony numbers in IRAIN-silenced U266 and RPMI-8226 cells exhibited a marked increase compared with si-NC-transfected cells (Fig. 2D and E). The data from the colony formation assay are presented in Fig. 2F.

Knockdown of IRAIN inhibits apoptosis. The effect of IRAIN knockdown on apoptosis was analyzed by flow cytometry. Compared with that in the si-NC group, the apoptosis of MM cells was inhibited in the si-IRAIN group. The apoptotic rates were $13.8 \%$ in the U266 group and $14.9 \%$ in the RPMI-8226 group (Fig. 3A and B). Compared with si-NC transfection, si-IRAIN transfection inhibited apoptosis in MM cells ( $\mathrm{P}<0.001$, Fig. 3C). 

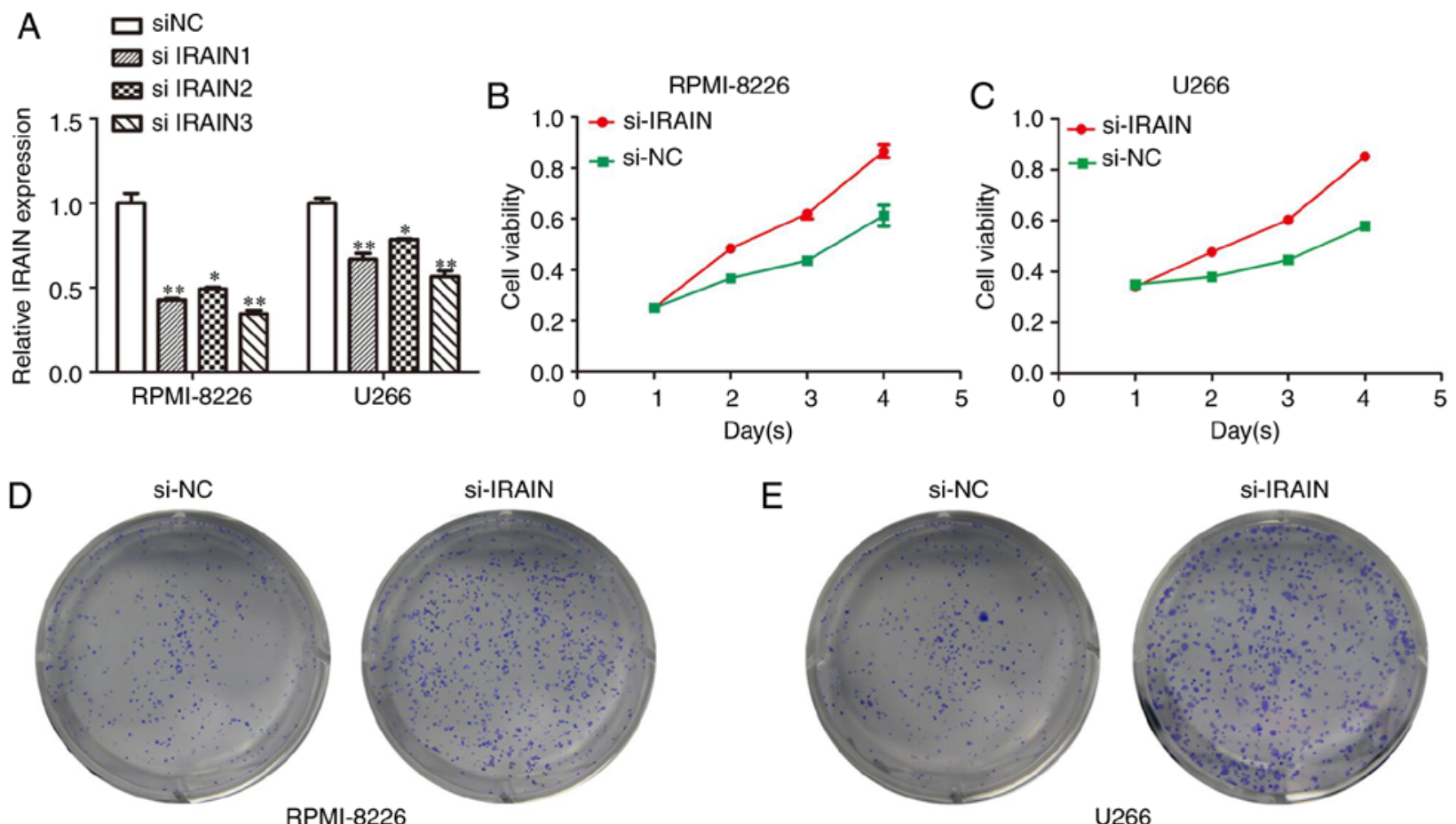

RPMI-8226

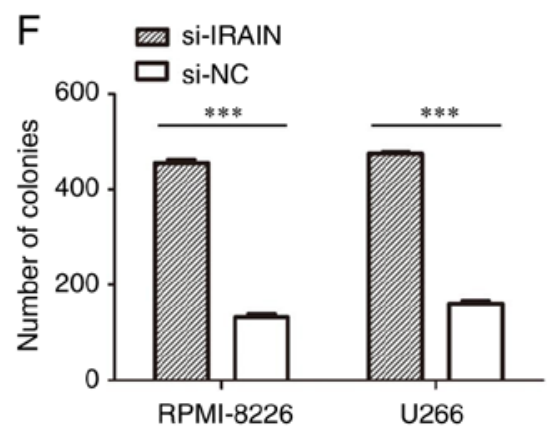

Figure 2. Regulatory effects of IRAIN on MM cell proliferation. (A) Expression levels of IRAIN determined by reverse transcription-quantitative polymerase chain reaction in RPMI-8226 and U266 cells transfected with si-NC, si-IRAIN1, si-IRAIN2 and si-IRAIN3. The Cell Counting Kit 8 assay demonstrated that (B) RPMI-8226 and (C) U266 cells transfected with si-IRAIN demonstrated increased cell viability compared with cells transfected with si-NC. Colony formation assays for (D) RPMI-8226 and (E) U266 cells transfected with si-NC or si-IRAIN. (F) Results from the colony formation assays. An increased number of colonies were observed for cells transfected with si-IRAIN compared with si-NC. Data represent the mean \pm standard deviation ( $\mathrm{n}=3$ ). ${ }^{*} \mathrm{P}<0.05$, ${ }^{* *} \mathrm{P}<0.01,{ }^{* * *} \mathrm{P}<0.001$ vs. si-NC. MM, multiple myeloma; IRAIN, IGF1R antisense imprinted non-protein coding RNA; si, small interfering; NC, negative control.

miR-125b reverses the effect of IRAIN on MM cell apoptosis. To further investigate whether IRAIN exerts biological functions through miR-125b, rescue experiments were performed by inhibiting miR-125b expression in si-IRAIN cells (U266). Flow cytometry demonstrated that cell apoptosis was reduced in si-IRAIN cells, whereas the addition of an miR-125b inhibitor partially reversed this effect (Fig. 4; $\mathrm{P}<0.01$ ).

IRAIN is a target of miR-125b. In the present study it was discovered that the tumor suppressor IRAIN was a target of miR-125b as predicted by miRcode (Fig. 5A). To determine whether miR-125b bound to the 3'-UTR of IRAIN, luciferase reporter assays were performed. As expected, miR-125b overexpression inhibited luciferase activity. By contrast, cells with mutant IRAIN 3'-UTRs displayed increased luciferase activity (Fig. 5B). The results also demonstrated that IRAIN knockdown increased miR-125b expression levels (Fig. 5C). At the same time, treatment with the miR-125b inhibitor enhanced IRAIN expression (Fig. 5D).

\section{Discussion}

MM is a neoplasm of terminally differentiated B cells (PCs), accounting for $\sim 0.8 \%$ of all new cancer cases (12). Dysregulation of IncRNA expression serves an important role in cancer development and lncRNAs are becoming potential prognostic biomarkers in cancer (13). An increasing number of studies have provided evidence to suggest that the dysregulation of IncRNAs may contribute to MM progression. For example, Sedlarikova et al (14) reported that IncRNA UCA1 was downregulated in MM. Furthermore, Cho et al (15) demonstrated that the IncRNA MALAT1 is overexpressed in $\mathrm{MM}$ and may serve as a marker to predict disease progression. However, the function and underlying mechanism of lncRNAs in MM remain unclear. Understanding the roles of IncRNAs as tumor suppressors or oncogenes may help to identify novel potential biomarkers for early diagnosis and new epigenetic molecular targets for MM patients. 
A

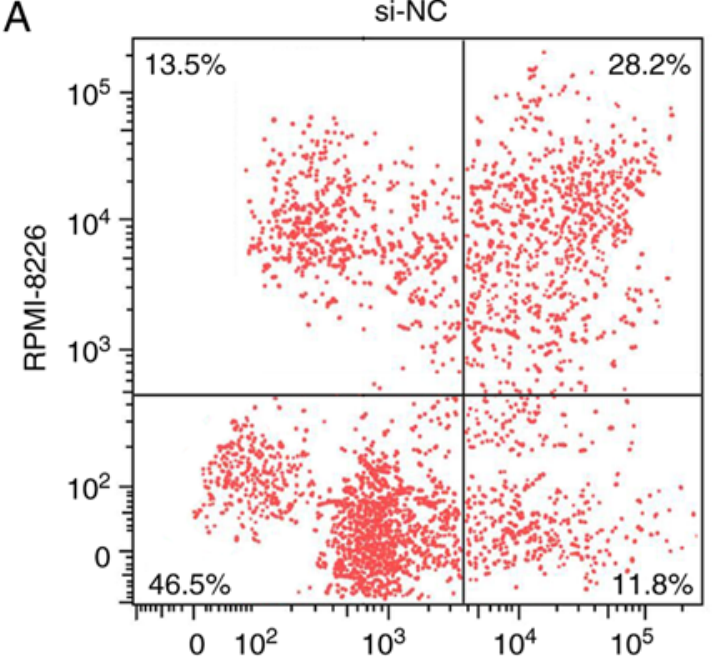

B

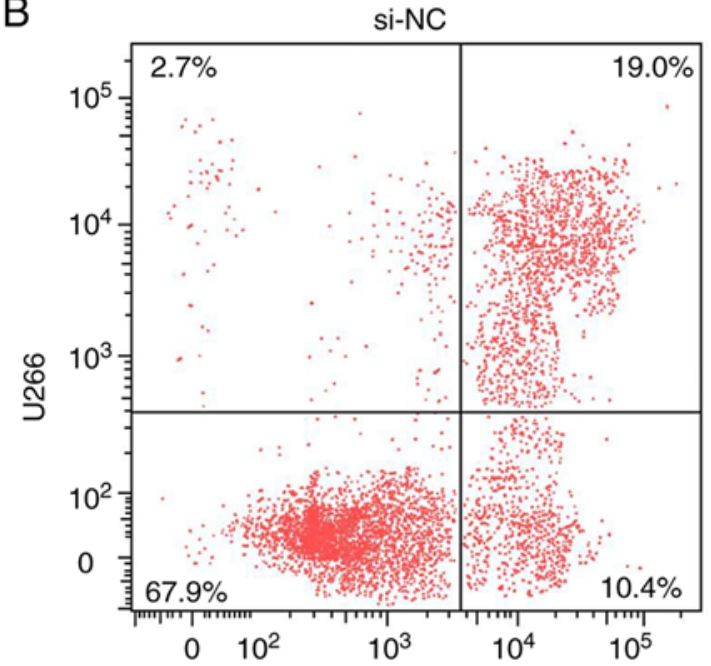

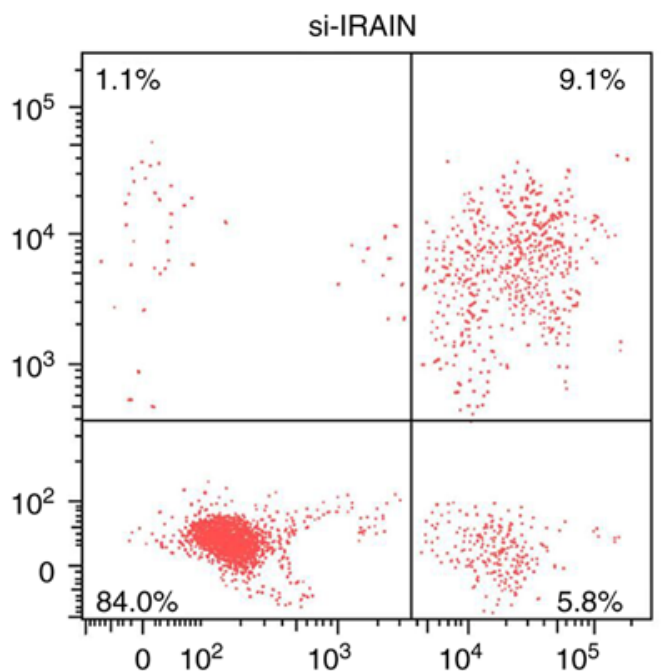

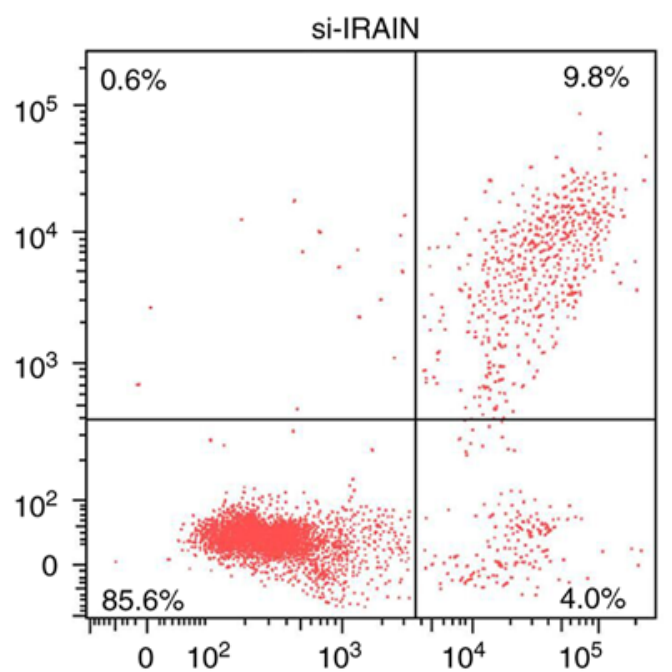

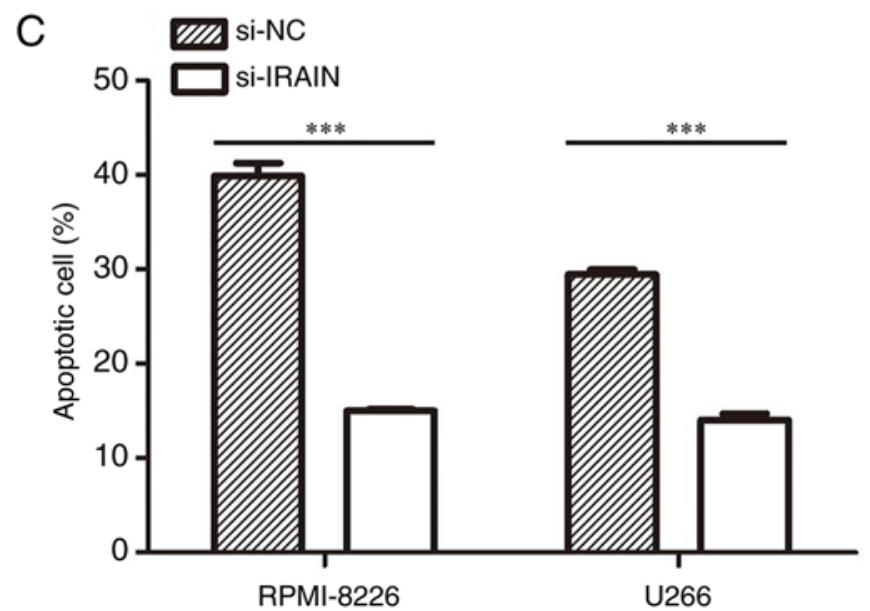

Figure 3. Effect of IRAIN expression on multiple myeloma cell apoptosis. Cell apoptosis was measured in (A) RPMI-8226 and (B) U266 cells by flow cytometry $24 \mathrm{~h}$ after transfection. (C) Apoptosis rates of the transfected cells in each group. Apoptosis was significantly lower in cells transfected with si-IRAIN compared with si-NC. Data represent the mean \pm standard deviation $(n=3) .{ }^{* * *} \mathrm{P}<0.001$. IRAIN, IGF1R antisense imprinted non-protein coding RNA; si, small interfering; $\mathrm{NC}$, negative control.

IRAIN is an antisense noncoding RNA that was first identified in hematopoietic malignancies, with a pattern of decreased expression in AML (9). Previous studies have indicated that IRAIN is associated with breast cancer (10), non-small cell lung cancer (16) and pancreatic cancer (17). However, there is limited evidence to suggest a link between IRAIN and MM.
Notably miR-125b is a member of the miR-17-92 cluster and has been demonstrated to function as an oncomir in numerous human cancer types. A study performed by Wang et al (18) demonstrated that miR-125b was highly expressed in breast cancer and Shen et al (19) reported that miR-125b expression was markedly increased in type 2 diabetes mellitus. Previous 

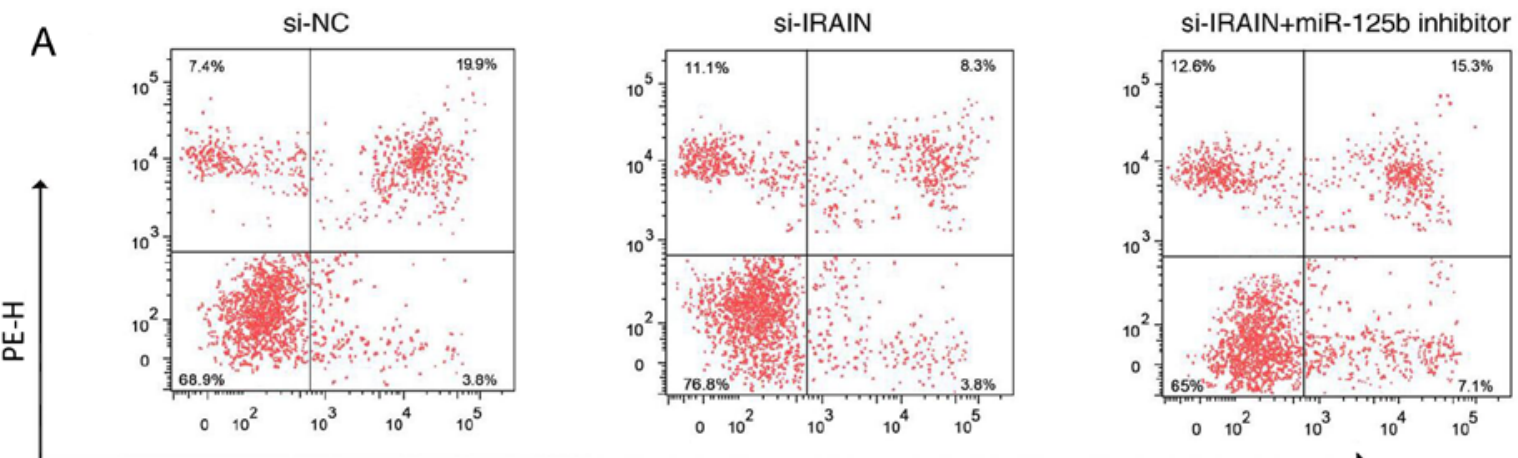

FITC-H

B

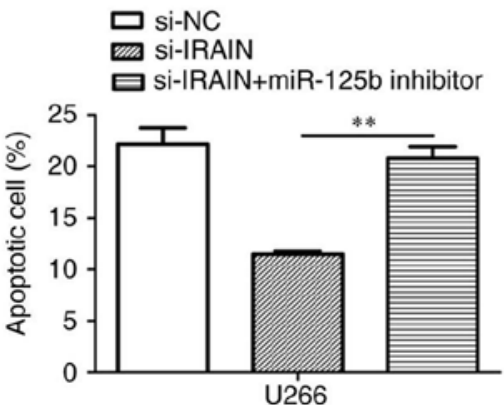

C

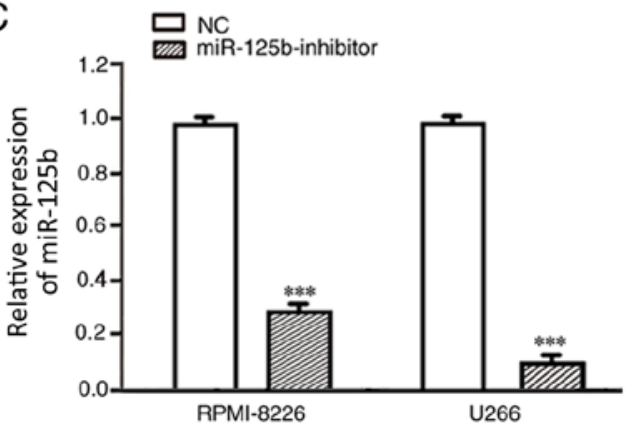

Figure 4. miR-125b reversed the effect of IRAIN on MM cell apoptosis. (A) Cell apoptosis of MM cells was detected by flow cytometry. (B) Apoptosis rates of the transfected cells in each group. Cells transfected with si-IRAIN in combination with a miR-125b inhibitor displayed enhanced apoptosis compared with cells transfected with si-IRAIN only. (C) Expression levels of miR-125b determined by reverse transcription-quantitative polymerase chain reaction in RPMI-8226 and U266 cells transfected with NC or miR-125b inhibitor. ${ }^{* *} \mathrm{P}<0.01$ and ${ }^{* * *} \mathrm{P}<0.001$. miR, microRNA; MM, multiple myeloma; IRAIN, IGF1R antisense imprinted non-protein coding RNA; si, small interfering; NC, negative control.

A IRAIN WT miR-125b

IRAIN MUT

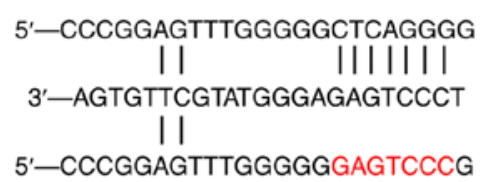

C

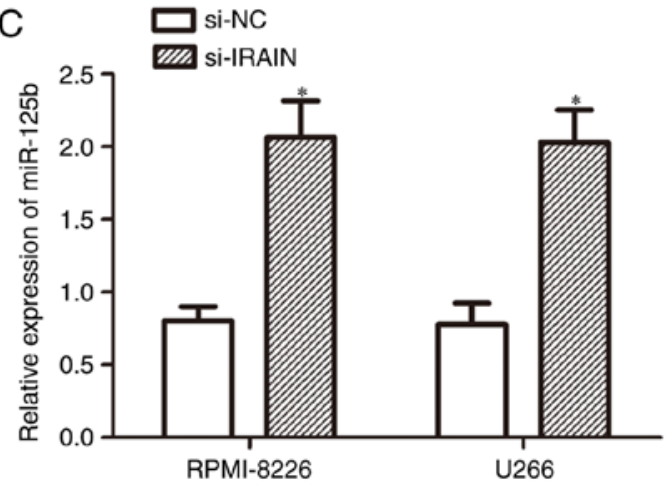

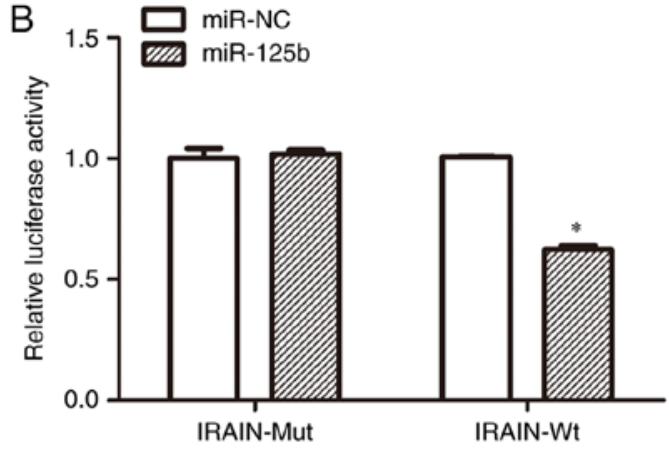

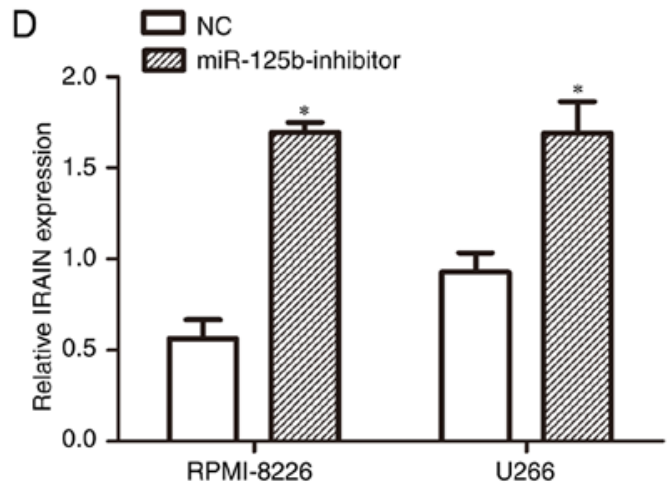

Figure 5. Identification of IRAIN as a target of miR-125b. (A) Schematic of miR-125b binding to IRAIN-WT 3'-UTR. (B) Relative luciferase activity detected by dual-luciferase reporter gene activity assay. Relative luciferase activity is significantly lower for IRAIN-WT compared with IRAIN-Mut. "P<0.05 vs. miR-NC. (C) Expression levels of miR-125b following IRAIN siRNA transfection measured using RT-qPCR in RPMI-8226 cells and U266 cells. Expression levels are significantly higher in cells transfected with si-IRAIN compared with si-NC. ${ }^{\mathrm{P}}<0.05$ vs. si-NC. (D) Expression levels of IRAIN following transfection with the miR-125b inhibitor, measured using RT-qPCR in RPMI-8226 and U266 cells. IRAIN expression levels were significantly increased in the presence of the miR-125b-inhibitor compared with NC. "P<0.05 vs. respective NC group. IRAIN, IGF1R antisense imprinted non-protein coding RNA; miR, microRNA; UTR, untranslated region; WT, wild-type; Mut, mutant; siRNA, small interfering RNA; RT-qPCR, reverse transcription-quantitative polymerase chain reaction; $\mathrm{NC}$, negative control. 
research has also demonstrated that high levels of miR-125b was associated with shortened progression-free survival times (20). In the current study, it was demonstrated that IRAIN expression was substantially downregulated in MM plasma and cell lines. Furthermore, an increased level of miR-125b expression in plasma from samples was observed. It was demonstrated that miR-125b was also upregulated in MM cell lines. IRAIN depletion promoted MM cell proliferation and led to the inhibition of MM cell apoptosis. Furthermore, the present study reported that IRAIN was a direct target of miR-125b. These data suggest that IRAIN may be a tumor suppressor lncRNA and that the upregulation of miR-125b in MM may be associated with the development and progression of the disease.

Increasing evidence suggests that IncRNAs may act as endogenous miRNA sponges by binding to miRNAs $(21,22)$. Zhang et al (23) demonstrated that lncRNA UCA1 promoted cancer progression by acting as a competing endogenous RNA of activating transcription factor 2 in prostate cancer. Additionally, Xia et al (24) demonstrated that lncRNA Fer-1 like family member 4 suppressed cancer cell growth by acting as a competing endogenous RNA and regulating phosphatase and tensin homolog expression. However, further investigations are required to determine whether the relationship between IRAIN and miR-125b is similar in MM.

In summary, the present study demonstrated that IRAIN may act as a tumor suppressor in MM, whilst miR-125b may act as a tumor promoter. A negative correlation between IRAIN and miR-125b was observed in MM plasma. Downregulation of IRAIN significantly increased the expression of miR-125b. Silencing of IRAIN promoted cell growth and inhibited cell apoptosis, and miR-125b reversed the effect of IRAIN on MM cell apoptosis. Furthermore, by using dual-luciferase reporter assays, IRAIN was identified as a target of miR-125b. Knockdown of IRAIN suppressed MM cell proliferation in vitro. Therefore, the present study highlights the importance of the miRNA-lncRNA interaction in tumorigenesis. Consequently IRAIN may be used to predict the clinical prognosis of MM patients and may be a novel therapeutic target for treating MM.

\section{Acknowledgements}

Not applicable.

\section{Funding}

The present study was supported by the International Collaboration Fund from the National Science and Technology Committee of China (grant no. 2011DFA32820), Innovation Fund Project in Jiangxi Province (grant no. YC2016-B018), and the National Natural Science Fund Project (grant no. 81460037).

\section{Availability of data and materials}

The datasets used and/or analyzed during the current study are available from the corresponding author on reasonable request.

\section{Authors' contributions}

YJ performed the experiments, analyzed the data and wrote the manuscript. JC performed flow cytometry. GC conceived and designed the experiments. All authors read and approved the final manuscript.

\section{Ethics approval and consent to participate}

The Research Ethics Committee of Nanchang University (Nanchang, China) approved this study and written informed consent was obtained from all study subjects.

\section{Patient consent for publication}

Not applicable.

\section{Competing interests}

The authors declare that they have no competing interests.

\section{References}

1. Sonneveld P, Avet-Loiseau H, Lonial S, Usmani S, Siegel D, Anderson KC, Chng WJ, Moreau P, Attal M, Kyle RA, et al: Treatment of multiple myeloma with high-risk cytogenetics: A consensus of the International Myeloma Working Group. Blood 127: 2955-2962, 2016.

2. Mimura N, Hideshima T and Anderson KC: Novel therapeutic strategies for multiple myeloma. Exp Hematol 43: 732-741, 2015.

3. Gao JR, Qin XJ, Jiang H, Gao YC, Guo MF and Jiang NN: Potential role of lncRNAs in contributing to pathogenesis of chronic glomerulonephritis based on microarray data. Gene 643: 46-54, 2018.

4. Duguang L, Jin H, Xiaowei Q, Peng X, Xiaodong W, Zhennan L, Jianjun Q and Jie Y: The involvement of lncRNAs in the development and progression of pancreatic cancer. Cancer Biol Ther 18: 927-936, 2017.

5. Meng YB, He X, Huang YF, Wu QN, Zhou YC and Hao DJ. Long noncoding RNA CRNDE promotes multiple myeloma cell growth by suppressing miR-451. Oncol Res 25: 1207-1214, 2017.

6. Li B, Shi C, Zhao J and Li B: Long noncoding RNA CCAT1 functions as a ceRNA to antagonize the effect of miR-410 on the down-regulation of ITPKB in human HCT-116 and HCT-8 cells. Oncotarget 8: 92855-92863, 2017.

7. Gu Y, Xiao X and Yang S: LncRNA MALAT1 acts as an oncogene in multiple myeloma through sponging miR-509-5p to modulate FOXP1 expression. Oncotarget 8: 101984-101993, 2017.

8. Niu XB, Fu GB, Wang L, Ge X, Liu WT, Wen YY, Sun HR, Liu LZ, Wang ZJ and Jiang BH: Insulin-like growth factor-I induces chemoresistence to docetaxel by inhibiting miR-143 in human prostate cancer. Oncotarget 8: 107157-107166, 2017.

9. Sun J, Li W, Sun Y, Yu D, Wen X, Wang H, Cui J, Wang G, Hoffman AR and Hu JF: A novel antisense long noncoding RNA within the IGF1R gene locus is imprinted in hematopoietic malignancies. Nucleic Acids Res 42: 9588-9601, 2014.

10. Kang L, Sun J, Wen X, Cui J, Wang G, Hoffman AR, Hu JF and Li W: Aberrant allele-switch imprinting of a novel IGF1R intragenic antisense non-coding RNA in breast cancers. Eur J Cancer 51: 260-270, 2015.

11. Livak KJ and Schmittgen TD: Analysis of relative gene expression data using real-time quantitative PCR and the 2(-Delta Delta C(T)) method. Methods 25: 402-408, 2001.

12. Merz M, Jauch A, Hielscher T, Bochtler T, Schönland SO, Seckinger A, Hose D, Bertsch U, Neben K, Raab MS, et al: Prognostic significance of cytogenetic heterogeneity in patients with newly diagnosed multiple myeloma. Blood Adv 2: 1-9, 2017.

13. Shen X, Zhang Y, Wu X, Guo Y, Shi W, Qi J, Cong H, Wang X, Wu X and Ju S: Upregulated lncRNA-PCAT1 is closely related to clinical diagnosis of multiple myeloma as a predictive biomarker in serum. Cancer Biomark 18: 257-263, 2017.

14. Sedlarikova L, Gromesova B, Kubaczkova V, Radova L, Filipova J, Jarkovsky J, Brozova L, Velichova R, Almasi M, Penka M, et al: Deregulated expression of long non-coding RNA UCA1 in multiple myeloma. Eur J Haematol 99: 223-233, 2017. 
15. Cho SF, Chang YC, Chang CS, Lin SF, Liu YC, Hsiao HH, Chang JG and Liu TC: MALAT1 long non-coding RNA is overexpressed in multiple myeloma and may serve as a marker to predict disease progression. BMC Cancer 14: 809, 2014.

16. Feng J, Sun Y, Zhang EB, Lu XY, Jin SD and Guo RH: A novel long noncoding RNA IRAIN regulates cell proliferation in non small cell lung cancer. Int J Clin Exp Pathol 8: 12268-12275, 2015.

17. Lian Y, Wang J, Feng J, Ding J, Ma Z, Li J, Peng P, De W and Wang K: Long non-coding RNA IRAIN suppresses apoptosis and promotes proliferation by binding to LSD1 and EZH2 in pancreatic cancer. Tumour Biol 37: 14929-14937, 2016.

18. Wang H, Tan G, Dong L, Cheng L, Li K, Wang Z and Luo H: Circulating MiR-125b as a marker predicting chemoresistance in breast cancer. PLoS One 7: e34210, 2012.

19. Shen Y, Xu H, Pan X, Wu W, Wang H, Yan L, Zhang M, Liu X, $\mathrm{Xia} S$ and Shao Q: miR-34a and miR-125b are upregulated in peripheral blood mononuclear cells from patients with type 2 diabetes mellitus. Exp Ther Med 14: 5589-5596, 2017.
20. Piatopoulou D, Avgeris M, Marmarinos A, Xagorari M, Baka M, Doganis D, Kossiva L, Scorilas A and Gourgiotis D: miR-125b predicts childhood acute lymphoblastic leukaemia poor response to BFM chemotherapy treatment. Br J Cancer 117: 801-812, 2017.

21. Li F, Huang C, Li Q and Wu X: Construction and comprehensive analysis for dysregulated long non-coding RNA (lncRNA)-associated competing endogenous RNA (ceRNA) network in gastric cancer. Med Sci Monit 24: 37-49, 2018.

22. Zhao Y, Wang $\mathrm{H}$, Wu C, Yan M, Wu H, Wang J, Yang $\mathrm{X}$ and Shao Q: Construction and investigation of IncRNA-associated ceRNA regulatory network in papillary thyroid cancer. Oncol Rep 39: 1197-1206, 2018.

23. Zhang S, Dong X, Ji T, Chen G and Shan L: Long non-coding RNA UCA1 promotes cell progression by acting as a competing endogenous RNA of ATF2 in prostate cancer. Am J Transl Res 9: 366-375, 2017

24. Xia T, Liao Q, Jiang X, Shao Y, Xiao B, Xi Y and Guo J: Long noncoding RNA associated-competing endogenous RNAs in gastric cancer. Sci Rep 4: 6088, 2014. 